\title{
AN UNCOMMON CASE OF SPONTANEOUS RESOLUTION OF MURAL THROMBUS CAUSING SYMPTOMATIC SEVERE STENOSIS OF THE EXTRACRANIAL PART OF THE INTERNAL CAROTID ARTERY
}

\author{
Milan Labonek ${ }^{\mathrm{a} *}$, Daniel Sanak ${ }^{\mathrm{a}}$, Roman Herzig ${ }^{\mathrm{a}}$, Stanislav Burval ${ }^{\mathrm{b}}$, Petr Bachledac, \\ Ivanka Vlachova ${ }^{\mathrm{a}}$, Petr Hlustik ${ }^{\mathrm{a}, \mathrm{b}}$, Petr Kanovsky ${ }^{\mathrm{a}}$
}

\begin{abstract}
Stroke Center, Departments of ${ }^{a}$ Neurology and ${ }^{b}$ Radiology, ${ }^{c} I I^{\text {nd }}$ Surgical Department, University Hospital, Olomouc, Czech Republic

e-mail:naklo@email.cz
\end{abstract}

Received: November 1, 2006; Accepted: November 20, 2006

Key words: Severe carotid stenosis/Spontaneous resolution/Ultrasonography/Digital subtraction angiography/Carotid endarterectomy

Objectives: Etiology of ischemic stroke(IS) remains sometimes unexplained in younger subjects. Its well-known causes are atherosclerosis(AS), embolization, arterial dissection, congenital defects, vasculitides, smoking, use of oral contraceptives, coagulopathy and arterial dissection, or vasospasm.

Case report: A 54-year old male subject was examined because of motor disturbance in his left upper extremity. Computerized tomography(CT) of the brain did not detect any focal pathological changes. Utrasonographic(USG) examination revealed severe stenosis of the right internal carotid artery(ICA) caused by hypoechogenic material, the blood velocity beyond the stenosis was $240 \mathrm{~cm} / \mathrm{s}$. The USG finding was confirmed by digital subtraction angiography (DSA). Based on the results of these examinations, an acute carotid endarterectomy (CEA) was indicated. During CEA the vascular surgeon observed patency of the ICA with only small calcified AS plaque on its posterior side. Ultrasonographic examination, performed on the $5^{\text {th }}$ day, showed a small calcified AS plaque on the posterior side of the right ICA.

Conclusion: In conclusion, if we exclude the possibility of a false positivity of 2 imaging methods at once, we have encountered a very unusual case of SR of stenosing material in the extracranial part of the ICA, initially causing its severe stenosis manifesting by acute neurological symptomatology. The mechanism of this SR remains unclear and the problem should be further studied, preferably using less invasive AG methods.

\section{INTRODUCTION}

Etiology of ischemic stroke (IS) remains sometimes unexplained in younger subjects. Its well-known causes are atherosclerosis (AS), embolization, arterial dissection, congenital defects, vasculitides, smoking, use of oral contraceptives, coagulopathy and, in some cases, arterial dissection, or vasospasm.

Based on the results of the authors' own randomized prospective trial ${ }^{1}$, an urgent disobliteration of the internal carotid artery (ICA) has been used at the Stroke Center, Department of Neurology, University Hospital, Olomouc, Czech Republic since 1998 in carefully selected patients suffering from an acute IS caused by a recent ICA occlusion. An urgent carotid endarterectomy (CEA) is performed in severe (> $70 \%$ ) ICA stenosis in selected acute IS patients at this center, as well.

The authors describe an unusual case of spontaneous resolution (SR) of mural thrombus causing symptomatic severe stenosis of the ICA in its extracranial part.

\section{CASE REPORT}

A 54-year old male suffered from rheumatic fever in his childhood and from axial hiatal hernia. In his medical history he had fracture of fibula on the right side and he also underwent repeated surgery of venous varices in his legs. He was in the past a smoker (20 years ago), he drank spirits on a regular every day basis and, occasionally also beer and wine. His other medical history was negative, including cardiogenic and thromboembolic diseases.

His father died because of occupational injury. His mother underwent heart valve replacement surgery and died of pulmonary embolism. No other coagulation disorders were present in his family history.

He was examined at the Stroke Center, Department of Neurology, University Hospital, Olomouc, Czech Republic because of motor disturbance in his left upper extremity, lasting for 30 hours. He noticed a deficit of problem with his left thumb and index finger movement with initial slowness. These problems occurred for the first time in his life.

On the examination, light drop of the left upper extremity in Mingazzini, positive Dufour and Russecki signs 
on the left, limitation of abduction, adduction and flexion of the left index finger and increased brachial and styloradial deep tendon reflexes on the left side were observed. No disorder of sensation was noted.

Computerized tomography (CT) of the brain did not detect any focal pathological changes (Fig. 1). Ultrasonographic (USG) examination revealed severe (75\% according to the North American Symptomatic Carotid Endarterectomy Trial criteria ${ }^{2}$ stenosis of the right ICA caused by hypoechogenic material. Transcranial color-coded ultrasonography and bilateral USG examination of periorbital circulation demonstrated normal findings. Basic haematological and biochemical tests did not show any pathological findings either. The USG finding was confirmed by digital subtraction angiography (DSA), performed by the Seldinger technique, using non-ionic contrast iohoxol Omnipaque ${ }^{\circledR} 240$ (Amersham Health A/S, Oslo, Norway). An irregular defect was present in filling of the right ICA bulb along its posterior side with a conical reduction of the arterial lumen. In its upstream section, the ICA was adequately wide, its outlines were smooth and the carotid siphon had standard shape (Fig. 2). No pathological changes were present in the periphery of the rights ICA territory.

Based on the results of these examinations, acute CEA was indicated. During CEA, performed 90 minutes after the DSA using a routine incision of the ICA, the vascular surgeon observed patency of the ICA with only small calcified AS plaque on its posterior side. The surgeon closed the artery in a standard way using a $\operatorname{Goretex}^{\circledR}(W$. L. Gore

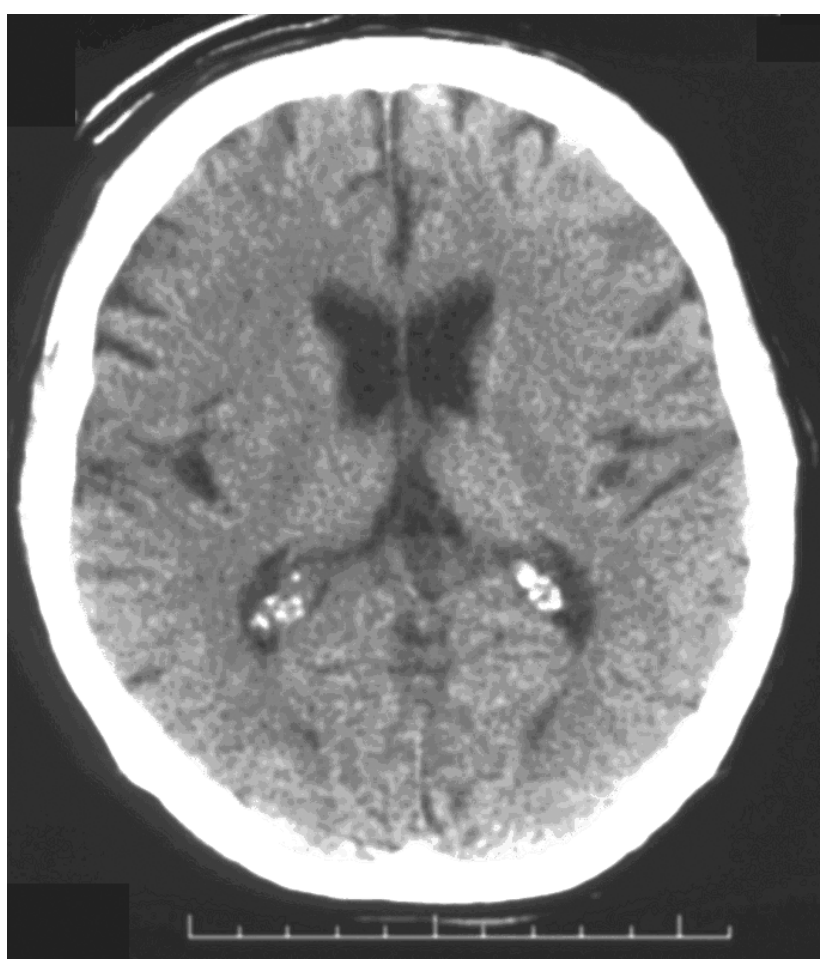

Fig. 1. Brain transversal native CT scan - showing no pathology
\& Associates, Inc. Medical Products Division Flagstaff, Arizona, USA) patch.

No progress of the neurological symptomatology was observed during the following days. A light paresis of left hand, with partial recovery, was still present on the $3^{\text {rd }}$ day after the surgery. Ultrasonographic examination, performed on the $5^{\text {th }}$ day, showed a small calcified AS plaque on the posterior side of the right ICA, as observed during the CEA.

The patient started rehabilitation of his left arm. Transesophageal echocardiography did not detect intracardial thrombus. Hematological tests including thrombosis markers (proteins $\mathrm{C}$ and $\mathrm{S}$, aPC-resistance, $\alpha$-2-antiplasmine, plasminogene, antithrombine III, factor II, cardiolipine and anti-lupus antibodies), performed one month later, failed to confirm any congenital thromboembolic predisposition. Only von Willebrandt factor (vWF) $284 \%$, tissue plasminogene activator (TPA) $14.8 \mathrm{ng} / \mathrm{ml}$, plasminogen-activator inhibitor 1 (PAI-1) $101 \mathrm{ng} / \mathrm{ml}$ and

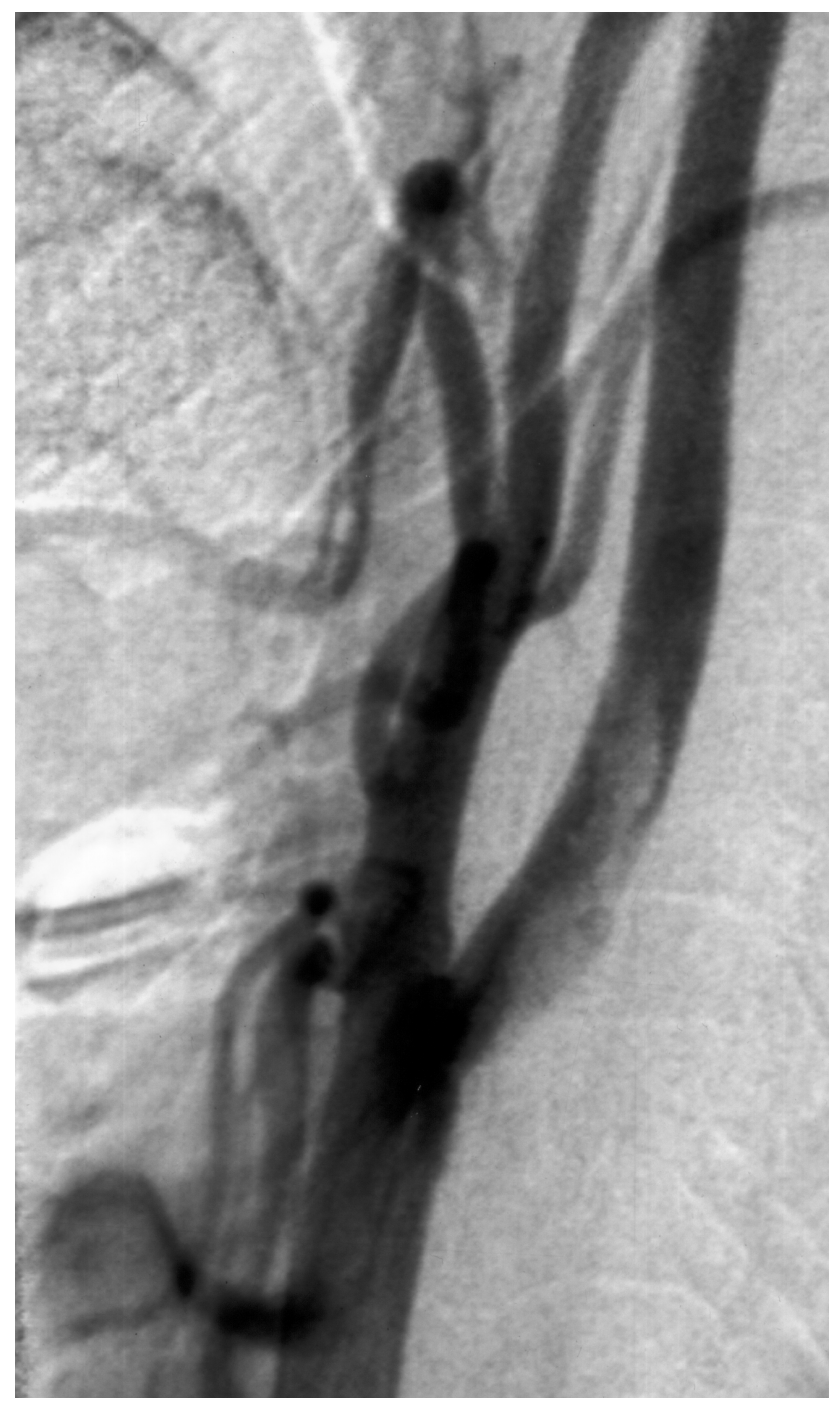

Fig. 2. DSA - right ICA - irregular defect in the bulb along its posterior side with a conical reduction of the arterial lumen and with normal finding beyond the bulb. 
factor VIII ( $154 \%$ ) were elevated. The lipid spectrum and serum homocysteine were normal.

A hyperintense lesion $(15 \times 9 \mathrm{~mm})$ localized in the right parietal lobe subcortically was found in T2-weighted images magnetic resonance imaging (MRI) (Fig. 3). This lesion, possibly of postischemic etiology, did not exhibit contrast enhancement after Prohance ${ }^{\circledR}$ (Altana Pharma AG, Konstanz, Germany) administration and had no correlation in T1-weighted images.

Three months after the stroke, only a light motor deficit was present in the patient's left hand, without any other clinical problems. He was treated with standard antithrombotic therapy - acetylsalicylic acid (Anopyrin ${ }^{\circledR}$ ) tablets $100 \mathrm{mg}$ a day. Repeated USG examination showed a stable finding of a small calcified AS plaque on the posterior side of the right ICA, causing light (20\%) stenosis with a normal finding in other arteries.

Transthoracic and transesophageal echocardiography, performed one month later showed normal findings, without the presence of thrombus and without any other cardiac pathology. Six months after the onset of neurological symptoms, he underwent surgery with polypectomy of apedunculated sigmoid polyp and 2 years later he was diagnosed with microcytary sideropenic anemia caused by hemorrhoidal bleeding.

\section{DISCUSSION}

In 1958, Lehrer documented for the first time the possibility of SR of the occluded cerebral arteries in angiographic (AG) series ${ }^{3}$. Since then, this has been quite a common finding in the case of cerebral arteries.

However, the cases of SR of a previously occluded ICA have been reported only anecdotally in its supraclinoid segment. Also, in patients treated with thrombolytic therapy, ICA occlusions have been shown to be more resistant to intravenous recombinant tissue plasminogen activator (rt-PA) than those located in the middle cerebral artery ${ }^{4}$. However, a recent prospective study using carotid duplex USG showed recanalization of six lesions in the followup of 18 patients suffering from an acute ICA occlusion thus, it is possible this phenomenon is more common than previously supposed ${ }^{5}$.

This aside, we have found only a few cases of ICA SR at its origin or in its siphon, demonstrated by USG or AG, in the literature ${ }^{5-12}$.

So far to date, no reliable data have been published on the incidence and clinical consequences of SR of the extracranial ICA occlusion ${ }^{6}$. Its frequency has been reported within a wide range of $17 \%$ to $67 \%$, increasing with the length of interval between occlusion onset and follow-up $\mathrm{AG}^{5,7}$. Higher ICA SR incidence (up to $86 \%$ ) has also reported in cases caused by spontaneous arterial dissection ${ }^{10}$.

The exact SR timing remains unclear. For example, Fieschi and Bozzao reported in 1969 the SR of distal ICA occlusion on follow-up AG performed 2 to 3 weeks after its initial presentation ${ }^{13}$. More recently, the occurrence of very early SR, within 1 to 2 hours of onset, has been also reported $^{7}$. Equally early SR, within 90 minutes of last verification of severe ICA stenosis by DSA, was present also in our case. On the other hand, in the case reported by Manganaro et al., ICA SR, documented by AG, occurred after several months 9 .

The SR mechanism, whether it occurs early or late, also remains unclear ${ }^{7}$. This problem is strongly associated with the etiology of the detected transient severe ICA stenosis. Several mechanisms, including vasospasm, distal embolization of occlusive clot, and spontaneous clot lysis, have been proposed as an SR explanation.

In our case, transient severe ICA stenosis could have been caused by an acute thrombus accruing onto an older calcified AS plaque at the dorsal side and dissolving completely within the time from the DSA performance to the surgical opening of the ICA. The dissolution could have been accelerated by Heparin bolus (5000 units) administered intravenously before ICA opening. Two cases of spontaneous and heparin-induced partial recanalization of the ICA occlusion were documented by Schelp et al., using both USG and DSA. Residual high-grade stenosis at the ICA origin was successfully removed surgically in both cases $^{14}$.

There is also a possibility of an embolism of a small part of the thrombus, appearing hypoechogenic at the USG, to the arterial periphery, but no worsening of the neurological symptomatology was observed following the surgery. The small T2-weighted MRI hyperintense lesion, possibly of postischemic etiology, found in the right parietal lobe, could correlate to the clinical symptomatology caused by the severe right ICA stenosis and likely lasting for 30 hours, and also a correlate of the artery-to-artery embolism of a small part of the AS plaque. Embolism of the whole thrombus to the arterial periphery is impossible, considering its size. Hematological and biochemical tests did not confirm any congenital thromboembolic predisposition. The finding of $\mathrm{vWF}$ and PAI-1 increased levels 1 month later correlate with a nonspecific endothelial lesion.

Manganaro et al. suggested, that the activation of endothelial spontaneous antithrombotic mechanisms may allow dissolution of a thrombus, once the cause of the

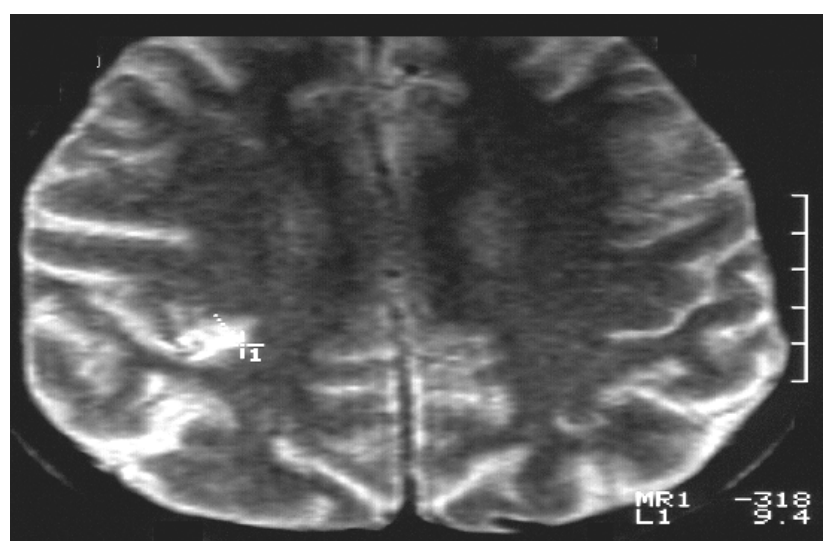

Fig. 3. Brain transversal T2-weighted MRI - hyperintensive lesion $(15 \times 9 \mathrm{~mm})$ in the right parietal lobe subcortically. 
thrombosis has been identified (the use of oral contraceptives containing high levels of estrogens in their case) and removed and when the endothelium had resumed its functional integrity 9 .

Another possible ICA SR explanation, for example in the case reported by Nguyen-Huynh et al., is that the vessel was not completely occluded and that the picture of the "occlusion", as seen on CT angiography (CTA), was a false positive finding resulting from extremely slow blood flow. To minimize this possibility, all angiographic runs should be carried out to the completion of the late venous phase through the distal extracranial ICA and through the entire intracranial ICA in cases of possible occlusion? While using the DSA, a "gold standard" of angiographic imaging, for confirmation of severe ICA stenosis in our patient, the possibility of false positive result seems to be lower compared to previously mentioned CTA imaging. The ICA was documented only in 2 AG projections and that is why the possibility of the artifact caused by an unequal diffusion of contrast matter should be taken into consideration. However, the possibility of false positive DSA results seems to be lower due to preceding the DSA, the same finding of a severe ICA stenosis was independently confirmed by 2 physicians using USG. Of course, in the case of USG, the assessment of stenosis degree can be relative and operator dependent.

However, regardless of the underlying mechanism, diagnosing recanalization is potentially important in establishing prognosis, assessing current treatment, and determining the future course of treatment?

SR of the acute ICA occlusion is an important phenomenon, the natural history and incidence of which have been so far only incompletely studied. The choice of AG methods, which should be used for its verification, should be discussed.

Conventional catheter AG remains the "gold standard" for distinguishing total arterial occlusion from hairline residual lumen. The use of this invasive technique is associated with up to $6.9 \%$ overall incidence of neurologic deficit, persisting in up to $0.52 \%$. This led to the search for less invasive methods for the evaluation of carotid artery stenosis. CTA is a rapid, safe, and minimally invasive method that has been shown to be highly sensitive and specific in diagnosing severe carotid stenosis and occlusion, including the results of the study previously performed at our department ${ }^{15}$. For example, CTA axial images have been found to have a sensitivity of $100 \%$, a specificity of $98 \%$, and an accuracy of $99 \%$ for diagnosing ICA occlusion. Moreover, preliminary studies have demonstrated the high accuracy of CTA in distinguishing hairline residual ICA lumen ("string sign") from a true total vascular occlusion - more sensitively than either unenhanced MRI angiography or USG. Thus, CTA can be used for serial monitoring of anticoagulated patients with new-onset ICA occlusion for potential SR?

In conclusion, if we exclude the possibility of the false positivity of 2 imaging methods at once, we have encountered a very unusual case of SR of stenosing material in the extracranial part of the ICA, initially causing severe stenosis manifesting as acute neurogical symptomato- logy. The mechanism of this SR remains unclear and the problem should be further studied, preferably using less invasive AG methods. The ability to conveniently assess ICA patency, for example by CTA, may not only influence management of individual patients but also could help us better establish the true incidence of ICA SR in future studies.

\section{ACKNOWLEDGEMENTS}

Supported by the IGA Ministry of Health CR grants number NR/7830-3/2004 and NR/8579-3/2005.

\section{REFERENCES}

1. Herzig R, Bar M, Vlachova I, Krupka B, Sanak D, Bachleda P, Utikal P, Machac J, Burval S, Mares J. Ischaemic stroke due to the acute internal carotid artery occlusion: Results of the emergent carotid disobliteration versus conservative treatment [abstract]. Cerebrovasc Dis 2003; 16(Suppl 4):57-58.

2. North American Symptomatic Carotid Endarterectomy Trial Collaborators. Beneficial effect of carotid endarterectomy in symptomatic patients with high-grade carotid stenosis. N Engl J Med. 1991; 325:445-453.

3. Lehrer GM. Arteriographic demonstration of collateral circulation in cerebrovascular disease. Neurology 1958; 8:27-32.

4. Bezerra DC, Reichhart MD, Maulaz AB, Wintermark M, Michel P, Maeder P, Meuli R, Bougousslavsky J. Carotid occlusion reopening and subsequent outcome after iv-rtPA therapy within 3 hours of stroke [abstract]. Neurology 2005; 64 (Suppl 1):A263-A264.

5. Calleja S, De la Vega V, Llaneza JM, Lopez-Roger R, Gutierrez JM, Lahoz CH. Spontaneous recanalization of acute internal carotid artery occlusion. Ann Vasc Surg 2004; 18:490-492.

6. Camporese G, Verlato F, Salmistraro G, Ragazzi R, Andreozzi GM. Spontaneous recanalization of internal carotid artery occlusion evaluated with color flow imaging and contrast arteriography. Int Angiol 2003; 22:64-71.

7. Nguyen-Huynh MN, Lev MH, Rordorf G. Spontaneous recanalization of internal carotid artery occlusion. Stroke 2003; 34:10321034.

8. Meves SH, Muhs A, Federlein J, Buttner T, Przuntek H, Postert T. Recanalization of acute symptomatic occlusions of the internal carotid artery. J Neurol 2002; 249:188-92.

9. Manganaro A, Ruggeri M, Ando G, Longo M, Vita G. Endothelial functions in pathophysiology of thrombosis and fibrinolysis: late spontaneous recanalization of an occluded internal carotid artery - a case report. Angiology 2002; 53:99-103.

10. Markwalder TM, Starrett RW, Mumenthaler M. Spontaneous bilateral recanalization in bilateral internal carotid artery occlusion. Stroke 1980; 11:95-98.

11. Sindermann F, Brugel R, Giedke H. Spontaneous recanalization of internal carotid artery occlusions Neuroradiology 1974; 7, 53-56.

12. Hoshino, H, Takagi, M, Takeuchi, I. Recanalization of intracranial carotid occlusion detected by duplex carotid sonography. Stroke 1989; 20:680-686.

13. Fieschi C, Bozzao L. Transient embolic occlusion of the middle cerebral and internal carotid arteries in cerebral apoplexy. J Neurol Neurosurg Psychiatry 1969; 32:236-40.

14. Schelp R, Bickel A, Kolominski P, Schweiger H. Spontaneous and heparin-induced recanalisation of the completly occlused internal carotid artery - a neglect phenomenon? Fortschr Neurol Psychiatr 1995; 63:277-282.

15. Herzig R, Burval S, Krupka B, Vlachova I, Urbanek K, Mares J. Comparison of ultrasonography, CT angiography, and digital subtraction angiography in severe carotid stenoses. Eur J Neurol 2004; 11:774-781. 